\title{
AKT is translocated to the mitochondria during etoposide-induced apoptosis of HeLa cells
}

\author{
BYOUNGDUCK PARK ${ }^{1}$, YOUNG-TAE $\mathrm{JE}^{2}$ and KWANG-HOON CHUN ${ }^{3}$ \\ ${ }^{1}$ College of Pharmacy, Keimyung University, Daegu 42601; ${ }^{2}$ College of Pharmacy and Research Institute of Pharmaceutical Sciences, \\ Seoul National University, Seoul 08826; ${ }^{3}$ Gachon Institute of Pharmaceutical Sciences, \\ College of Pharmacy, Gachon University, Incheon 21936, Republic of Korea
}

Received December 6, 2014; Accepted September 3, 2015

DOI: $10.3892 / \mathrm{mmr} .2015 .4378$

\begin{abstract}
Akt, or protein kinase B, is a key serine-threonine kinase, which exerts anti-apoptotic effects and promotes cell proliferation in response to various stimuli. Recently, however, it was demonstrated that Akt exhibits a proapoptotic role in certain contexts. During etoposide-induced apoptosis of HeLa cells, Akt enhances the interaction of second mitochondria-derived activator of caspases/direct IAP binding protein with low pI (Smac/DIABLO) and X-linked inhibitor of apoptosis protein by phosphorylating Smac at serine 67, and thus promotes apoptosis. However, the detailed mechanisms underlying Akt regulation in etoposide-mediated apoptosis remain to be determined. The present study investigated whether etoposide triggers the translocation of Akt into the mitochondria. It was found that Akt activity was increased and sustained during apoptosis triggered by etoposide in HeLa cells. During apoptosis, Akt was translocated from the cytoplasm into the mitochondria in a phosphoinositide 3-kinase-dependent manner at the early and late stages of apoptosis. Concomitantly, the depletion of Akt in the nuclear fraction was observed after etoposide treatment from analysis of confocal microscopy. The results suggest that etoposide-stimulated Akt is translocated into the mitochondria, thereby possibly enhancing its interaction with Smac and promoting apoptosis in HeLa cells. These results indicate that Akt may be a promising candidate for a pro-apoptotic approach in cancer treatment.
\end{abstract}

\section{Introduction}

Apoptosis, also termed programmed cell death, is involved in development, the elimination of damaged cells, and the maintenance of cell homeostasis. Deregulation of apoptosis may cause diseases, such as cancer, immune disorders and degenerative

Correspondence to: Dr Kwang-Hoon Chun, Gachon Institute of Pharmaceutical Sciences, College of Pharmacy, Gachon University, 191 Hambakmoero, Yeonsu-gu, Incheon 21936, Republic of Korea E-mail:khchun@gachon.ac.kr

Key words: Akt, mitochondria, translocation diseases (1). Apoptosis is an important cellular response to irreparable damage from exposure to external stimuli (2). Apoptosis can be induced by two different pathways; the extrinsic pathway, which involves signaling from death receptors, such as tumor necrosis factor receptor 1 (TNFR1), and the intrinsic pathway, which centers around the mitochondria (3). In recent years, it has been increasingly recognized that the mitochondria is pivotal in the early events of apoptosis. A key process in the mitochondrial apoptotic pathway is the release of a group of proteins, such as cytochrome $c$, second mitochondria-derived activator of caspases/direct IAP binding protein with low pI (Smac/DIABLO), apoptosis-inducing factor (AIF) and endonuclease $\mathrm{G}$ from the mitochondrial intermembrane space, which subsequently triggers a cascade of cytoplasmic changes (4).

Protein function associated with apoptosis can be modulated by post-translational modification, such as acetylation, phosphorylation and nitrosylation. Currently, numerous studies suggest that the function of various mitochondrial factors is regulated by the interplay between kinases and phosphatases, and thereby promote or inhibit apoptosis (5-7). As a pro-apoptotic kinase, c-Jun NH2-terminal kinase is associated with Smac/DIABLO release from the mitochondria into the cytoplasm during apoptosis in multiple myeloma cells (8). A complex of $\mathrm{c}-\mathrm{Abl}$ and protein kinase $\mathrm{C} \delta$ migrates into the mitochondria in response to various oxidative stresses, and induces cytochrome $c$ release from the mitochondria (9-11). Lck activity is required for mitochondrial-dependent apoptosis following irradiation and ceramide exposure $(12,13)$.

By contrast, cumulative studies have suggested that Akt inhibits apoptosis by inactivating Bad, Caspase-9 and Forkhead box (FOX) proteins by direct phosphorylation (14-16). Akt mediates cell proliferation, survival, migration, and glucose homeostasis via the canonical phosphoinositide 3-kinase (PI3K)-Akt signaling pathway (17). Downstream targets of Akt for survival signaling include transcription factor cAMP-response element binding protein or I $\kappa \mathrm{B}$ kinases that regulate the activity of nuclear factor $-\kappa \mathrm{B}$ (18). Furthermore, Akt activation is sufficient to inhibit the release of cytochrome $c$ from the mitochondria and the change in inner mitochondrial membrane potential (19). Recent data, however, indicated that Akt can act as a pro-apoptotic regulator under specific conditions (20). Numerous studies have demonstrated that Akt sensitized cells to oxidative stress-mediated 
apoptosis $(21,22)$ while pharmacological inhibition of Akt reduced apoptosis mediated by various cytotoxic or apoptotic intracellular molecules (23-26). The pro-apoptotic effect of Akt was also demonstrated and it was shown that Akt phosphorylates Smac/DIABLO and enhances its interaction with XIAP in etoposide-induced apoptosis (27). Although increasing evidence indicates that Akt has dual roles in the determination of cell fate in a context-dependent way, the molecular mechanisms are not yet well established.

In the present study, whether Akt is activated and translocated into the mitochondria during etoposide induced apoptosis was investigated. This appears to be a key step in the progression of apoptotic, and regulation of this step may provide effective as a novel strategy in the treatment of cancer in humans.

\section{Materials and methods}

Materials. Etoposide, $\mathrm{KCl}, \mathrm{MgCl}_{2}$, EDTA, EGTA, DTT and PMSF was purchased from Sigma-Aldrich (St. Louis, MO, USA). LY294002 was obtained from Calbiochem (San Diego, CA, USA). Dulbecco's modified Eagle's medium (DMEM), antibiotics-antimycotics, HEPES and fetal bovine serum (FBS) were purchased from Invitrogen Life Technologies (Grand Island, NY, USA). Protease inhibitor cocktail was purchased from Roche (Nutley, NJ, USA). Antibodies against Akt (rabbit monoclonal IgG; cat. no. 4691; Cell Signaling Technology, Inc., Danvers, MA, USA), phospho-Akt (Ser473; rabbit ant-human monoclonal IgG; cat. no. 4060; Cell Signaling Technology, Inc), $\alpha$-tubulin (rabbit polyclonal ant-human IgG; cat. no. 2144; Cell Signaling Technology, Inc.) and cytochrome $c$ oxidase subunit IV (COX IV; rabbit monoclonal IgG; cat. no. 4850; Cell Signaling Technology, Inc.) were purchased from Cell Signaling Technology Inc. (Beverly, MA, USA). Anti-poly ADP-ribose polymerase (PARP) antibody (rabbit polyclonal anti-human IgG; cat. no. sc-7150; Santa Cruz Biotechnology, Inc., Santa Cruz, CA, USA) was obtained from Santa Cruz Biotechnology Inc. (Santa Cruz, CA, USA). All primary antibodies were diluted into 1:1,000 for western blotting and 1:100 for confocal imaging. Secondary antibodies were diluted 1:5,000 for western blotting and 1:400 for confocal imaging.

Cell culture and induction of apoptosis. Human HeLa cervical carcinoma cells (Seoul National University Cancer Center, Seoul, Republic of Korea) were cultured in DMEM supplemented with $10 \%$ heat-inactivated FBS and antibiotics-antimycotics in a humidified incubator with $5 \% \mathrm{CO}_{2}$ at $37^{\circ} \mathrm{C}$. Apoptosis was induced by incubation with $10 \%$ FBS-supplemented DMEM containing $85 \mu 1$ etoposide. To inhibit the PI3K-Akt signaling pathway, the cells were pre-treated with LY294002, a PI3K inhibitor, for $1 \mathrm{~h}$ prior to incubation with the etoposide.

Subcellular fractionation. The etoposide-treated HeLa cells $\left(1 \times 10^{5}\right)$ were collected and resuspended in buffer, containing $20 \mathrm{mM}$ HEPES (pH 7.4), $10 \mathrm{mM} \mathrm{KCl}, 1.5 \mathrm{mM} \mathrm{MgCl}{ }_{2} 1 \mathrm{mM}$ EDTA, $1 \mathrm{mM}$ EGTA, $1 \mathrm{mM}$ DTT, $1 \mathrm{mM}$ PMS and protease inhibitor cocktail. Following incubation on ice for $15 \mathrm{~min}$, the cells were homogenized and centrifuged at $1,000 \mathrm{x} \mathrm{g}$ for $10 \mathrm{~min}$ at $4^{\circ} \mathrm{C}$. The supernatant was then centrifuged at $100,000 \mathrm{x} \mathrm{g}$ for $1 \mathrm{~h}$ at $4^{\circ} \mathrm{C}$. The supernatant (cytosol fraction) was then collected and the lysates (mitochondrial fraction) were lysed using lysis buffer.

Immunoblot analysis. Cells were lysed in lysis buffer containing $0.5 \%$ Triton X-100, $20 \mathrm{mM}$ Tris (pH 7.5); $2 \mathrm{mM} \mathrm{MgCl}_{2}$, $1 \mathrm{mM}$ DTT, $1 \mathrm{mM}$ EGTA, $50 \mathrm{mM} \beta$-glycerophosphate, $25 \mathrm{mM} \mathrm{NaF}$ and $1 \mathrm{mM} \mathrm{Na}_{3} \mathrm{VO}_{4}$, containing $1 \mathrm{mM}$ PMSF and protease inhibitor cocktail. The protein concentrations were determined using the MicroBCA ${ }^{\mathrm{TM}}$ Protein Assay reagents (Pierce Biotechnology, Inc., Rockford, IL, USA) according to the manufacturer's instructions. Cell lysates were resolved by SDS-PAGE (12\%) and transferred to polyvinylidene difluoride membranes (EMD Millipore, Billerica, MA, USA). Membranes were blocked with 5\% non-fat dry milk in TBST (TBS with $0.1 \%$ Tween-20; Sigma-Aldrich) buffer for $30 \mathrm{~min}$ at room temperature. The membranes were incubated with the specific primary antibodies in 5\% non-fat dry milk in TBST buffer $(1: 1,000)$. The bands were visualized with enhanced chemiluminescence (West-Zol plus ${ }^{\circledR}$; Intron Biotechnology, Sungnam, Republic of Korea).

Confocal microscopy. Cells on coverslips were washed twice with phosphate-buffered saline (PBS) and fixed on ice with 3\% paraformaldehyde/PBS for $10 \mathrm{~min}$, and then washed again with PBS. Residual paraformaldehyde was quenched by incubation with $0.1 \mathrm{M}$ glycine for $10 \mathrm{~min}$. After washing with PBS, cells were permeabilized with $0.1 \%$ Triton X-100 in PBS for 3 min, washed with PBS, and incubated in blocking solution (5\% milk) for $10 \mathrm{~min}$. Cells were incubated with anti-Akt and COX IV antibodies (1:100) in blocking buffer overnight at $4^{\circ} \mathrm{C}$. Nuclei were counterstained with 4',6-diamidino-2-phenylindole (DAPI). Subcellular localization of the proteins was visualized using a Nikon Eclipse Ti inverted microscope (Nikon, Tokyo, Japan) and analyzed using NIS-Elements software (ver. 4.0; Nikon Corporation, Tokyo, Japan). Pearson's correlation test was used to analyze the correlation between the expression levels of Akt and COX IV in immunocytochemistry using the NIS-Elements software.

\section{Results}

Akt translocates into the mitochondria during etoposide-induced apoptosis. Previously, the activation of Akt during etoposide-induced apoptosis in HeLa cells was reported (27). In the present study, etoposide-mediated activation of Akt by phosphorylation of the Ser473 residue in a time-dependent manner was confirmed (Fig. 1A and B). As an indicator of the induction of apoptosis, PARP cleavage was detected. As Akt activation is associated with Smac phosphorylation during apoptosis in HeLa cells, it was surmised that Akt activation is associated with the mitochondrial function during apoptosis. To test this hypothesis, the localization of Akt in etoposide-treated HeLa cells was examined by separating the mitochondrial fraction from the cytoplasmic fraction. As shown in Fig. 2A, the Akt level in the mitochondria increased significantly after the induction of apoptosis. The induction of Akt translocation at various time points during apoptosis was further confirmed. The Akt level in the mitochondria was increased significantly as early as $2 \mathrm{~h}$ after etoposide stimulation, which was further elevated with prolonged incubation by 
A

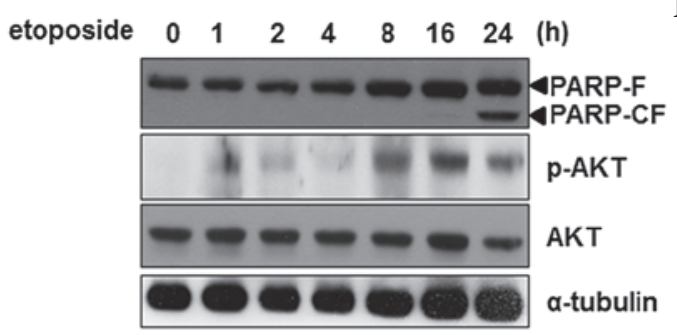

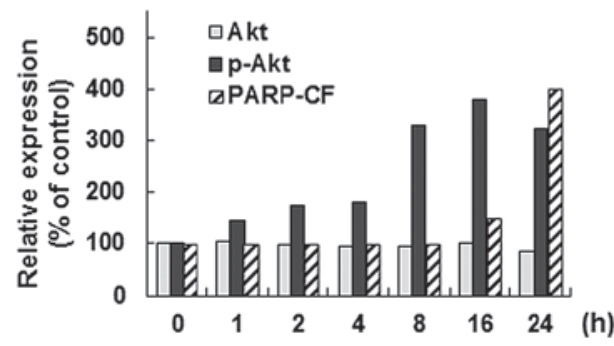

Figure 1. Activation of Akt in HeLa cells during etoposide-induced apoptosis. HeLa cells were treated with $85 \mu \mathrm{M}$ etoposide for various periods as indicated and cell lysates were subjected to SDS-PAGE. (A) The levels of PARP-F and PARP-CF of PARP, p-Akt, Akt were measured by immunoblot analysis. $\alpha$-tubulin was used as a loading control. (B) The level of phosphorylation of Akt was expressed as a bar graph and compared with the levels of Akt and PARP-CF. PARP, poly ADP-ribose polymerase; PARP-F, full length PARP; PARP-CF, cleaved form of PARP.

A

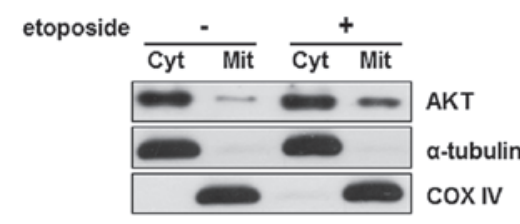

C

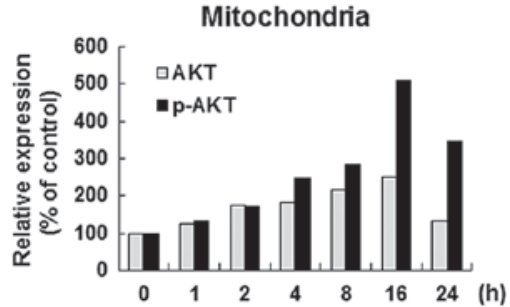

B etoposide $\quad 0 \begin{array}{llllllll}0 & 1 & 2 & 4 & 8 & 16 & 24 & \text { (h) }\end{array}$

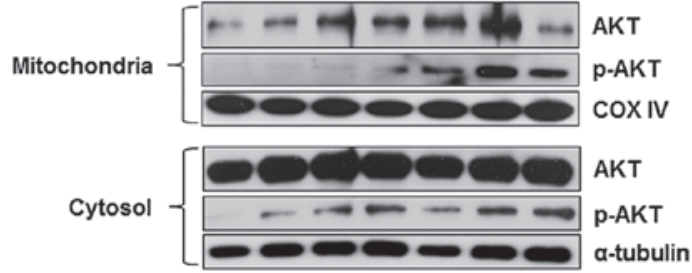

Cytosol

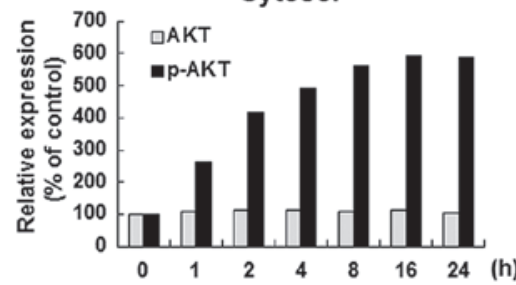

Figure 2. Mitochondrial and cytoplasmic fractionation analysis of Akt in etoposide-treated HeLa cells. (A) Etoposide-treated $(85 \mu \mathrm{M}, 16 \mathrm{~h})$ cells were fractionated into the mitochondrial and the cytoplasmic fractions. The level of phospho-Akt was measured using immunoblot analysis. $\alpha$-tubulin and COX IV were used as markers for the cytoplasmic and mitochondrial fractions respectively. (B) Cells were treated with etoposide (85 $\mu \mathrm{M})$ for various times as indicated and fractionated into the mitochondrial and the cytoplasmic fractions. The levels of phospho-Akt and Akt were measured using immunoblot analysis. (C) The levels of Akt and phospho-Akt were expressed as a bar graph. Cyt, cytoplasm; mit, mitochondria; COX IV, cytochrome $c$ oxidase subunit IV.

$\mathbf{A}$

Merged

Akt

CoxIV

control
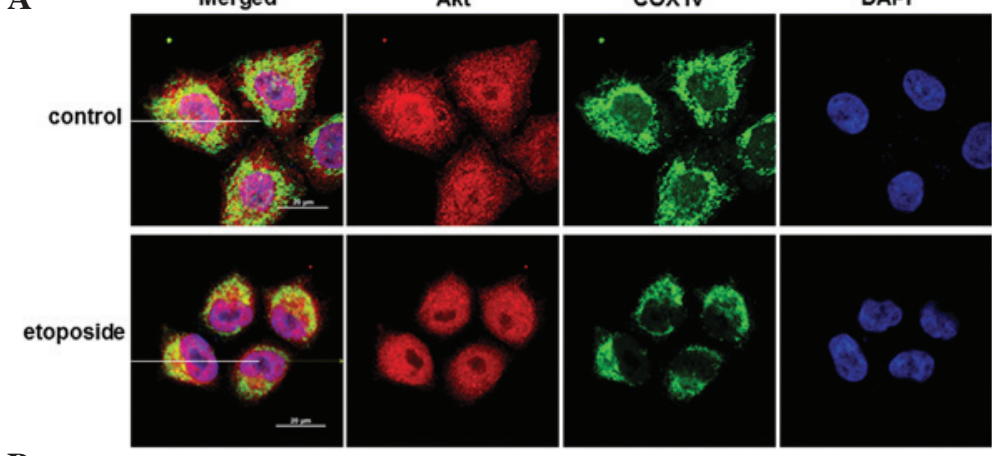

B

control

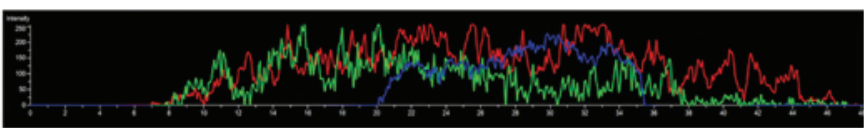

etoposide

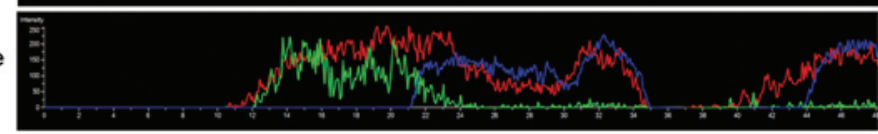

Figure 3. Confocal microscopic analysis of the subcellular localization of Akt during etoposide-induced apoptosis. (A) HeLa cells were incubated with or without etoposide $(85 \mu \mathrm{M})$ for $4 \mathrm{~h}$ and then co-stained with anti-Akt and anti-COX IV antibodies. Nuclei were counterstained with DAPI. To identify the subcellular prevalence of Akt in the mitochondria and the nuclei, each image was merged together. (B) To evaluate the effect of etoposide on the migration of Akt between subcellular compartments, the relative quantities of Akt and COX IV across the cell were determined by sectioning the cell perpendicularly, as shown by the white bar in (A). COX IV, cytochrome $c$ oxidase subunit IV; DAPI, 4',6-diamidino-2-phenylindole. 


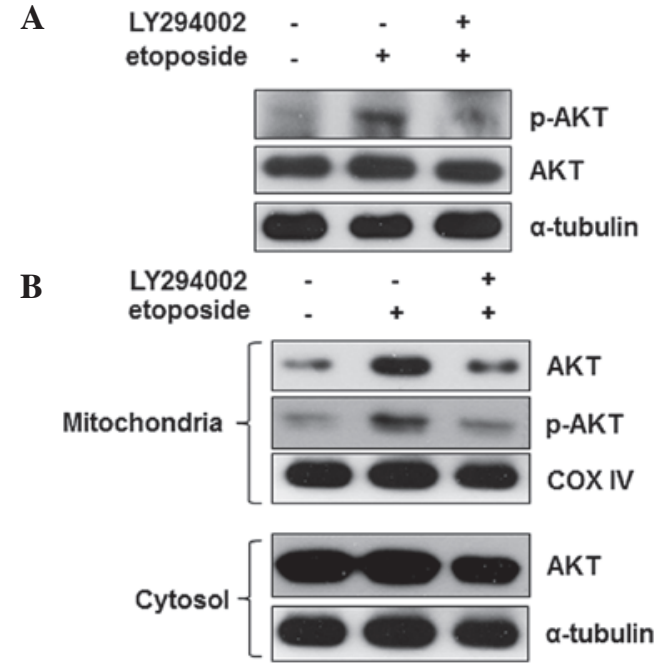

Figure 4. Effect of LY294002, a PI3K inhibitor, on the activity and migration of Akt during apoptosis. HeLa cells were pre-treated with LY294002 $(50 \mu \mathrm{M})$ for $1 \mathrm{~h}$ prior to incubation with etoposide for $18 \mathrm{~h}$. (A) The levels of Akt and phospho-Akt were measured by immunoblot analysis. $\alpha$-tubulin was used as a loading control. (B) Cells were separated into the cytosolic and mitochondrial fractions. The levels of Akt and phospho-Akt were determined by immunoblot analysis. $\alpha$-tubulin and COX IV were used as subcellular organelle markers for the cytoplasmic and mitochondrial fractions respectively. PI3K, phosphoinsoitide 3 kinase; COX IV, cytochrome $c$ oxidase subunit IV.

2.5-fold at $16 \mathrm{~h}$ (Fig. 2B upper panel). Similarly, the level of phosphorylated Akt, the active form, was also increased by etoposide in the mitochondria (Fig. 2B upper panel). However, the change in the expression level of Akt of the cytosol was not significant while clear induction of the p-Akt level was detected by etoposide (Fig. 2B lower panel).

Nuclear Akt levels are reduced during etoposide-induced apoptosis. To confirm the mitochondrial migration of $\mathrm{Akt}$ during etoposide-induced apoptosis, the subcellular localization of Akt was examined using confocal microscopy. Akt was distributed in the nucleus and the cytosol, and the partial co-localization of Akt and COX IV, a mitochondrial marker, was identified in control cells (Fig. 3A, upper). When cells are treated with etoposide, cell size was reduced by $\sim 50 \%$ (data not shown) by the shrinkage of the cytoplasm. The co-localization of Akt and COX IV was also detected in apoptosis-induced conditions (Fig. 3A, lower). However, a significant change in the parameter showing the levels of co-localization between etoposide-treated cells and control was not identified. The Pearson's correlation coefficient (PCC) was almost the same $\left(\mathrm{PCC}_{\text {Control }}=0.37 ; \mathrm{n}=7\right.$ cells; $\mathrm{SD}=0.082$ and $\mathrm{PCC}_{\text {Etoposide }}=0.37$ $; n=12$ cells; $\mathrm{SD}=0.092)$. This lack of change in the PCC value suggests the possibility that Akt migration into the mitochondria would be a local event during apoptosis, as the majority were located in other areas, including the cytosol. To further characterize the change in Akt distribution during apoptosis, levels of Akt, COX IV and DAPI were measured across a sectional plane (Fig. 3A, white bar) in etoposidetreated cells. When cells were sectioned along the cells (white bar), Akt (red) was located abundantly in the cytosol of cells undergoing apoptosis, while depletion of Akt was observed in the nucleolus suggesting that nuclear Akt was also affected by apoptotic signals induced by etoposide (Fig. 3B). The change in the subcellular distribution of Akt following etoposide treatment suggests that Akt may exhibit compartment-specific roles during apoptosis.

Activation of Akt is required for mitochondrial migration during apoptosis. Numerous stimuli activate Akt via the PI3K-Akt signaling pathway. To identify whether the etoposide-mediated regulation of Akt is dependent on the canonical PI3K-Akt signaling pathway, cells were pre-treated with LY294002, an inhibitor of PI3K, and the effects on the activity of Akt were measured. When cells were pretreated with LY294002, the etoposide-mediated Akt phosphorylation was reduced to the basal level (Fig. 4A) indicating that PI3K is a key regulator of Akt activation during etoposide-induced apoptosis. Furthermore, the effect of inhibition of PI3K on Akt mitochondrial translocation during apoptosis was identified. The Akt level in the mitochondria was also reduced to the basal level by LY294002 (Fig. 4B). These data show that PI3K may be an upstream regulator of the etoposide-mediated Akt activation and mitochondrial translocation. These findings demonstrate that Akt translocates into the mitochondria during etoposide-induced apoptosis and PI3K activation is required for the activity and localization of Akt in HeLa cells. This suggest that Akt may act by regulating the mitochondrial factors associated with the progression of apoptosis.

\section{Discussion}

Previously, it was demonstrated that Akt is activated during etoposide-induced apoptosis in HeLa cells in which Akt phosphorylates Smac and activates caspase-3 to promote apoptosis. As Smac is a mitochondrial protein that promotes cytochrome $c$ release during apoptosis, it was suggested that Akt exhibits a role in the mitochondria in the process of apoptosis. In this study, it was found that the Akt level in the mitochondria is low in a healthy normal state, but was increased significantly by apoptotic stimuli (Fig. 2). This translocation was accompanied by the elevation of Akt activity as shown by increased phosphorylation. Furthermore, it was demonstrated that PI3K activity is involved in these events during apoptosis (Fig. 4). To the best of our knowledge, this is the first study to demonstrate the translocation of Akt into the mitochondria during apoptosis.

Additionally, confocal microscopy further demonstrated that Akt resides in the nucleus of HeLa cells under normal conditions; however, Akt is depleted by etoposide treatment (Fig. 3). In particular, the level of Akt within the nucleolus was significantly diminished by etoposide indicating that this migration is involved in the pro-apoptotic function of Akt. This decrease of Akt from the nucleus may be not a general event during apoptosis, considering previous evidence that Akt translocation into the nucleus has a pro-apoptotic effect in methotrexate and docetaxel-treated MCF7 breast cancer cells (23). Thus, it appears that etoposide regulates distinct molecular targets from methotrexate or docetaxel, although they require Akt activation to induce apoptosis.

Akt has been known to be a survival factor and also a tumor-promoting agent. However, recent studies have shown that Akt is able to exhibit a pro-apoptotic effect under diverse conditions, such as oxidative stress (hydrogen peroxide, arsenite) $(20,24)$, stimulation by cytokines [Fas ligand (Fas-L), 
tumor necrosis factor $\alpha(\mathrm{TNF} \alpha)](28,29)$, and cytotoxic chemicals (staurosporine, methotrexate, docetaxel, etoposide) $(25,28)$.

Although the mechanisms underlying Akt activation and its ability to elicit a pro-apoptotic effect has not been elucidated clearly, several studies have suggested plausible explanations. Nogueira et al (21) showed that Akt sensitizes cells to oxidative stress-induced apoptosis by increasing reactive oxygen species (ROS) production as well as by lowering the expression of the oxygen scavenging enzymes, such as manganese superoxide dismutase, catalase and sestrin 3. In C141 epidermal cells, Fas ligand (Fas-L) caused Akt activation through the generation of the hydroxyl radical (30). In arsenite-, hydrogen peroxide-, $\mathrm{TNF} \alpha$ - and staurosporine-induced apoptosis, Akt phosphorylates and inhibits glycogen synthase kinase $3 \beta$ [one of the first identified substrates of Akt (31)] and FoxO3a. Following inhibition of FoxO3a, expression of oxygen scavenging enzymes is suppressed. In addition, Akt can induce the expression of Fas receptor mRNA, thus sensitizing cells to Fas-L. Furthermore, nuclear Akt was suggested to phosphorylate cyclin-dependent kinase $2(\mathrm{Cdk} 2)$ in methotrexate and docetaxel-induced apoptosis, thereby sustaining the cytoplasmic location of Cdk2, which may be associated with $\mathrm{G} 2 / \mathrm{M}$ cell cycle arrest prior to apoptosis (25). Although a number of studies have shown that Akt exerts pro-apoptotic effects through the phosphorylation of target molecules, the function of Akt in mitochondrial events have not been addressed. The present study demonstrated the re-location of Akt among subcellular organelles, which suggests that Akt may be important in mitochondria and may be a promising target for cancer treatment.

\section{Acknowledgements}

This study was supported by the Basic Science Research Program through the National Research Foundation of Korea, funded by the Ministry of Education, Science and Technology (grant no. 2010-0025409).

\section{References}

1. Jiang $\mathrm{X}$ and Wang $\mathrm{X}$ : Cytochrome C-mediated apoptosis. Annu Rev Biochem 73: 87-106, 2004.

2. Green DR: Apoptotic pathways: Paper wraps stone blunts scissors. Cell 102: 1-4, 2000.

3. Khosravi-Far R and Esposti MD: Death receptor signals to mitochondria. Cancer Biol Ther 3: 1051-1057, 2004.

4. van Gurp M, Festjens N, van Loo G, Saelens X and Vandenabeele P: Mitochondrial intermembrane proteins in cell death. Biochem Biophys Res Commun 304: 487-497, 2003.

5. Thomson M: Evidence of undiscovered cell regulatory mechanisms: Phosphoproteins and protein kinases in mitochondria. Cell Mol Life Sci 59: 213-219, 2002.

6. Boland ML, Chourasia AH and Macleod KF: Mitochondrial dysfunction in cancer. Front Oncol 3: 292., 2003.

7. Yoo SH, Kim HY, Rho JH, Jeong SY, Yun J, Yun I, Park HT and Yoo YH: Targeted inhibition of mitochondrial Hsp90 induces mitochondrial elongation in Hep3B hepatocellular carcinoma cells undergoing apoptosis by increasing the ROS level. Int J Oncol. Sep, 2015 (Epub ahead of print).

8. Chauhan D, Li G, Hideshima T, Podar K, Mitsiades C, Mitsiades N, Munshi N, Kharbanda S and Anderson KC: JNK-dependent release of mitochondrial protein, Smac, during apoptosis in multiple myeloma (MM) cells. J Biol Chem 278: 17593-17596, 2003.

9. Ito Y, Pandey P, Mishra N, Kumar S, Narula N, Kharbanda S, Saxena S and Kufe D: Targeting of the c-Abl tyrosine kinase to mitochondria in endoplasmic reticulum stress-induced apoptosis. Mol Cell Biol 21: 6233-6242, 2001.
10. Kumar S, Bharti A, Mishra NC, Raina D, Kharbanda S, Saxena S and Kufe D: Targeting of the c-Abl tyrosine kinase to mitochondria in the necrotic cell death response to oxidative stress. J Biol Chem 276: 17281-17285, 2001 .

11. Qi X and Mochly-Rosen D: The PKCdelta-Abl complex communicates ER stress to the mitochondria-an essential step in subsequent apoptosis. J Cell Sci 121: 804-813, 2008.

12. Belka C, Marini P, Lepple-Wienhues A, Budach W, Jekle A, Los M, Lang F, Schulze-Osthoff K, Gulbins E and Bamberg M: The tyrosine kinase lck is required for CD95-independent caspase- 8 activation and apoptosis in response to ionizing radiation. Oncogene 18: 4983-4992, 1999.

13. Hur YG, Yun Y and Won J: Rosmarinic acid induces p56lck-dependent apoptosis in Jurkat and peripheral $\mathrm{T}$ cells via mitochondrial pathway independent from Fas/Fas ligand interaction. J Immunol 172: 79-87, 2004.

14. Datta SR, Dudek H, Tao X, Masters S, Fu H, Gotoh Y and Greenberg ME: Akt phosphorylation of BAD couples survival signals to the cell-intrinsic death machinery. Cell 91: 231-241, 1997.

15. Cardone MH, Roy N, Stennicke HR, Salvesen GS, Franke TF, Stanbridge E, Frisch S and Reed JC: Regulation of cell death protease caspase-9 by phosphorylation. Science 282: 1318-1321, 1998.

16. Brunet A, Bonni A, Zigmond MJ, Lin MZ, Juo P, Hu LS, Anderson MJ, Arden KC, Blenis J and Greenberg ME: Akt promotes cell survival by phosphorylating and inhibiting a Forkhead transcription factor. Cell 96: 857-868, 1999.

17. Vivanco I and Sawyers CL: The phosphatidylinositol 3-kinase AKT pathway in human cancer. Nat Rev Cancer 2: 489-501, 2002.

18. Nicholson KM and Anderson NG: The protein kinase B/Akt signalling pathway in human malignancy. Cell Signal 14: 381-395, 2002.

19. Kennedy SG, Kandel ES, Cross TK and Hay N: Akt/Protein kinase $\mathrm{B}$ inhibits cell death by preventing the release of cytochrome c from mitochondria. Mol Cell Biol 19: 5800-5810, 1999.

20. Benbrook DM and Masamha CP: The pro-survival function of Akt kinase can be overridden or altered to contribute to induction of apoptosis. Curr Cancer Drug Targets 11: 586-599, 2011.

21. Nogueira V, Park Y, Chen CC, Xu PZ, Chen ML, Tonic I, Unterman $\mathrm{T}$ and Hay $\mathrm{N}$ : Akt determines replicative senescence and oxidative or oncogenic premature senescence and sensitizes cells to oxidative apoptosis. Cancer Cell 14: 458-470, 2008.

22. van Gorp AG, Pomeranz KM, Birkenkamp KU, Hui RC, Lam EW and Coffer PJ: Chronic protein kinase B (PKB/c-akt) activation leads to apoptosis induced by oxidative stress-mediated Foxo3a transcriptional up-regulation. Cancer Res 66: 10760-10769, 2006.

23. Galvez-Peralta M, Flatten KS, Loegering DA, Peterson KL, Schneider PA,Erlichman Cand Kaufmann SH: Context-dependent antagonism between Akt inhibitors and topoisomerase poisons. Mol Pharmacol 85: 723-734, 2014.

24. Shack S, Wang XT, Kokkonen GC, Gorospe M, Longo DL and Holbrook NJ: Caveolin-induced activation of the phosphatidylinositol 3-kinase/Akt pathway increases arsenite cytotoxicity. Mol Cell Biol 23: 2407-2414, 2003.

25. Maddika S, Ande SR, Wiechec E, Hansen LL, Wesselborg S and Los M: Akt-mediated phosphorylation of CDK2 regulates its dual role in cell cycle progression and apoptosis. J Cell Sci 121: 979-988, 2008

26. Andrabi S, Gjoerup OV, Kean JA, Roberts TM and Schaffhausen B: Protein phosphatase $2 \mathrm{~A}$ regulates life and death decisions via Akt in a context-dependent manner. Proc Natl Acad Sci USA 104: 19011-19016, 2007.

27. Jeong CH, Chun KS, Kundu J and Park B: Phosphorylation of Smac by Akt promotes the caspase-3 activation during etoposide-induced apoptosis in HeLa cells. Mol Carcinog 54: 83-92, 2015.

28. Ono K, Iwanaga Y, Hirayama M, Kawamura T, Sowa N and Hasegawa K: Contribution of caveolin-1 alpha and Akt to TNF-alpha-induced cell death. Am J Physiol Lung Cell Mol Physiol 287: L201-L209, 2004.

29. Suhara T, Mano T, Oliveira BE and Walsh K: Phosphatidylinositol 3-kinase/Akt signaling controls endothelial cell sensitivity to Fas-mediated apoptosis via regulation of FLICE-inhibitory protein (FLIP). Circ Res 89: 13-19, 2001.

30. Lu B, Wang L, Stehlik C, Medan D, Huang C, Hu S, Chen F, Shi X and Rojanasakul Y: Phosphatidylinositol 3-kinase/Akt positively regulates Fas (CD95)-mediated apoptosis in epidermal Cl41 cells. J Immunol 176: 6785-6793, 2006.

31. Cross DA, Alessi DR, Cohen P, Andjelkovich M and Hemmings BA: Inhibition of glycogen synthase kinase-3 by insulin mediated by protein kinase B. Nature 378: 785-789, 1995. 